\title{
Optimizing outcomes of open thoracoabdominal aortic aneurysm repair
}

\begin{abstract}
Open thoracoabdominal aortic aneurysm (TAAA) repair is a high-risk surgery associated with significant morbidity and mortality. It can be difficult to accumulate experience managing open TAAA repairs due to the low volume of cases and the increasing popularity of endovascular approaches. It is important to review management strategies for these challenging cases as clinical situations remain for which an open repair is preferred. Recent advances now allow for distal aortic perfusion during an off-pump open thoraco abdominal aortic aneurysm repair. This approach is not without risks and a clear, team based, plan is essential for successful management. Key considerations include managing co-morbidities, spinal cord protection, prevention of ischemia (coronary, cerebral, visceral, and peripheral), renal protection, massive transfusion, rapid hemodynamic changes, acidosis, coagulopathy, one lung ventilation, and postoperative pain control. In this case report, we detail an offpump perioperative approach involving retrograde aortic perfusion, transesophageal echocardiography, and multiple spinal cord protective strategies which resulted in a favorable patient outcome.
\end{abstract}

Keywords: thoracic aorta, thoracoabdominal aortic aneurysm, retrograde aortic perfusion, temporary axillofemoral bypass, vascular anesthesiology, spinal cord protection
Volume 3 Issue 2 - 2015

\author{
Braden Dulong,' Prasad Jetty, ${ }^{2}$ Ashraf Fayad ${ }^{3}$ \\ 'Anesthesia Resident, University of Ottawa, Canada \\ ${ }^{2}$ Assistant Professor, Department of Surgery, Canada \\ ${ }^{3}$ Associate Professor, Department of Anesthesia, Canada
}

Correspondence: Braden Dulong, Department of Anesthesia, The University of Ottawa, University of Ottawa, Dept of Anesthesia, 1053 Carling Ave, B302, Ottawa, ON, K IY 4E9, Tel 613798 5555, Fax I 613761 5209, Email bdulong@toh.on.ca

Received: October 19,2015 | Published: October 26, 2015
Abbreviations: TAAA, thoraco abdominal aortic aneurysm; POD, post operative day; TEE, tran-sesophageal echocardiography; $\mathrm{CPB}$, cardiopulmonary bypass; Lvads, left ventricular assist devices

\section{Introduction}

Aneurysmal degeneration of the aorta is a significant cause of death in first world nations. Repair of descending aortic aneurysms presents unique challenges, which could be encountered by any anesthesiologist involved in the management of vascular cases. Core management approaches are controversial and variations exist between leading institutions. Options include cancelling the case (medical management), fenestrated endovascular aortic aneurysm repair, hybrid repair (combined open and endovascular approach), or open surgery. When open surgery is preferred options include clamp and sew, full cardiopulmonary bypass, partial bypass, or retrograde perfusion via a temporary shunt. Key considerations include managing co-morbidities, spinal cord protection, prevention of ischemia (coronary, cerebral, visceral, and peripheral), renal protection, massive transfusion, rapid hemodynamic changes, acidosis, coagulopathy, one lung ventilation, and postoperative pain control. This publication describes our management approach and expands upon the above considerations.

\section{Case description}

A 57 year old male patient with a Crawford type II thoracoabdominal aortic aneurysm presented for open surgical repair. The aneurysm extended from the left subclavian artery to the abdominal aorta and involved the renal arteries. A multi-disciplinary preoperative assessment found no evidence of end organ failure and the patient was deemed to be optimized for the procedure.

The patient was brought into the operating room and standard monitors were applied. A thoracic epidural was placed for postoperative pain management. A lumbar drain was then placed with the patient in the right lateral decubitus position. A right radial arterial line was inserted. The patient was induced using sufentanil, midazolam, propofol, and rocuronium. An $8.5 \mathrm{~mm}$ endotracheal tube was used with an Arndt bronchial blocker placed in the left main bronchus. The balloon was kept down until one-lung ventilation was required. An internal jugular Cordis and an 8.5 French rapid infusion catheter were established. Fast flow fluid warmers and emergency medications were primed. A transesophageal echocardiogram probe was introduced for hemodynamic monitoring and a femoral arterial line was placed to monitor retrograde perfusion pressure.

Under general anesthesia a tunnelled right axillofemoral artery bypass was performed using a $10 \mathrm{~mm}$ Gore-Tex graft. The patient was then placed in a partial right lateral decubitus position and the table was hyperextended. An incision extending from above the symphysis pubis through to the fourth rib provided surgical access for the extensive dissection. A $20 \mathrm{~cm}$ portion of the aorta, just distal to the left subclavian artery, was then isolated using two clamps. Visceral perfusion distal to the caudad clamp was provided by retrograde flow from the axillofemoral bypass and monitored using the femoral arterial line. A $28 \mathrm{~mm}$ Dacron tube graft was attached to the aorta in an end to end fashion. By sequentially clamping and unclamping progressively distal portions of the aorta, the graft was extended beyond the renal arteries. The celiac, superior mesenteric, renal, and three intercostal arteries (added using a separate Crawford patch technique) were incorporated into the graft. Following surgical closure the temporary axillofemoral bypass graft was removed. The patient was in the operating room for 11.5 hours.

\section{Discussion}

\section{Coagulopathy}

The optimal management of coagulation during thoracoabdominal aortic aneurysm repair is challenging. Massive transfusion, hypothermia, acidosis, and the fibrinolytic state induced by visceral 
ischemia all increase the risk of bleeding. At the same time, arterial cross-clamping results in stagnant blood and presents a significant risk of intra-arterial thrombus formation. The fibrinolytic state induced by supraceliac cross-clamping appears to occur secondary to increased release of tissue-type plasminogen activator from ischemic vascular tissues. ${ }^{1}$ In cardiac cases antifibrinolytics have been found to reduce the need for blood transfusions with occasional inconsistent concerns regarding worsened renal, neurological, and cardiac outcomes..$^{2-10}$ Tranexamic acid, has been shown to be effective in reducing the need for allogeneic blood transfusion during thoracoabdominal aortic aneurysm repair. For this case, one gram of tranexamic acid was given prior to thoracotomy followed by an infusion of $1 \mathrm{mg} / \mathrm{kg} / \mathrm{h}$ for the duration of the case. Postoperatively there was no neurologic deficit and minimal deterioration in renal function (of note mannitol $0.5 \mathrm{~g} / \mathrm{kg}$ was given prior to aortic cross-clamping for renal protection). Renal function returned to baseline within two days. Supraceliac crossclamping also reduces liver perfusion which may alter the coagulation cascade and has been shown to prolong the effect of heparin. ${ }^{11}$ For this case, heparin was used for the axillo femoral artery bypass then reversed with protamine. For the duration of supraceliac aortic crossclamping no heparin was used and point of care arterial blood gas and INR measurements were completed every 10 minutes. Fresh frozen plasma (total volume $3745 \mathrm{ml}$ ) was transfused as needed to target an INR of less than 1.6. Cryoprecipitate $(9 \mathrm{ml})$, platelets $(678 \mathrm{ml})$, Cell saver RBCs $(2022 \mathrm{ml})$, and allogeneic RBCs $(3633 \mathrm{ml})$ were replaced as needed. No arterial thrombotic complications were noted.

\section{Bicarbonate}

The use of sodium bicarbonate in the context of reversible lactic acidosis is controversial. Most experts agree that sodium bicarbonate therapy is reasonable in the context of lactic acidosis if the $\mathrm{PH}$ is below 7.1 and adequate ventilation can be maintained. ${ }^{12}$ The aim of bicarbonate therapy is to avoid severe acidosis which can be associated with reduced left ventricular contractility, arrhythmias, arterial vasodilation, enzymatic dysfunction, and impaired responsiveness to catecholamines. ${ }^{12}$ Unfortunately, the potential benefits do not come without risks which include increased pCO2, accelerated lactate generation, reduced ionized calcium, hypernatremia, and extracellular fluid expansion. ${ }^{13,14}$ Given the easy diffusion of $\mathrm{CO} 2$ across membranes, a paradoxical increase in intracellular acidosis has been reported with bicarbonate therapy. Additionally, some studies have reported a protective effect of acidosis and suggest that rapid correction of acidosis following ischemic insult may lead to increased cellular death. ${ }^{14}$ Finally, given the appropriate clinical context, there is some evidence to suggest an increased mortality with bicarbonate use. ${ }^{14}$ Thoracoabdominal aortic aneurysm repair inevitably results in significant visceral ischemia. Severe acidosis can occur rapidly particularly during aortic unclamping. For this case an aggressive prophylactic sodium bicarbonate infusion $(841 \mathrm{ml}$ of $8.4 \%$ sodium bicarbonate) was used. The lowest recorded $\mathrm{pH}$ was 7.27 . No adverse clinical impact was noted.

\section{Transesophageal echocardiography}

Transesophageal echocardiography (TEE) is a minimally invasive monitor that provides unparalleled real time monitoring of cardiovascular function. Throughout this case, TEE was used to assess cardiac function (ejection fraction, regional wall motion abnormalities, and diastolic parameters), delineate the extent of disease (in particular to rule out proximal aortic dissection), monitor fluid status, monitor for ischemic changes, guide surgical interventions, and assess the status of surgical repair. Intraoperative acute diastolic dysfunction secondary to aortic cross clamping has been described and may carry significant morbidity. ${ }^{15}$ Intraoperative TEE is particularly useful for guiding fluid management. Left ventricular end diastolic volume estimated in the short-axis view at the mid papillary level provides a real time surrogate for volume status. ${ }^{16} \mathrm{~A}$ cardiac index $>2 \mathrm{~L} / \mathrm{min} /$ $\mathrm{m} 2$ was targeted and TEE played a key role in determining fluid and inotropic management.

\section{Spinal Cord Protection}

Spinal cord ischemia can occur during aortic cross-clamping or postoperatively due to the aortic graph partially occluding the blood supply to the spinal cord. Rates of paralysis of up to $40 \%$ have been reported in the literature. ${ }^{1}$ Numerous techniques have been used in an attempt to avoid this devastating complication including: temporary shunting, CSF drainage, epidural cooling, systemic cooling, local or systemic pharmacological interventions, spinal cord monitoring, and re-implantation of intercostal arteries. A 2012 Cochrane review stated that observational studies from experienced centres suggest a potential benefit for distal aortic perfusion, however, there are no randomized control trials investigating the use of distal aortic perfusion. ${ }^{17}$ For this case femoral arterial pressure was maintained with a target MAP between $70-100 \mathrm{mmHg}$. This target took into consideration maintaining spinal cord perfusion pressure and avoiding excessive hypertension which would increase bleeding. Options for distal aortic perfusion include cardiopulmonary bypass (CPB), left ventricular assist devices (LVADs), and temporary bypass grafts. CPB and LVADs may allow for more control over distal aortic perfusion but these techniques are not without risks. ${ }^{18}$ In particular, prevention of circuit thrombosis, the need for systemic heparinization, induced hemolytic/fibrinolytic states, and complications related to access sites must be considered.

While there are randomized control trials investigating the role of CSF drainage, the results are mixed. A 2012 Cochrane review concluded that there is limited data supporting the role of CSF drainage in thoracoabdominal aneurysm surgery based on three trials with a total of 287 patients. ${ }^{19}$ For this case a total of $177 \mathrm{ml}$ of CSF was drained during the 11.5 hour procedure. A CSF pressure of less than $10 \mathrm{mmHg}$ was targeted and the maximum rate of CSF drainage was kept below $20 \mathrm{ml} / \mathrm{h}$. At the end of the surgery the anesthetic was lightened briefly in order to confirm intact motor function in the legs.

\section{Postoperative course}

Postoperatively the patient was taken to the intensive care unit. $\mathrm{He}$ was extubated and weaned off vasopressors (target MAP 80 - 90mmHg) by postoperative day (POD) \#1. His lumbar drain was clamped and then discontinued on POD \#2. The epidural was removed on POD \#5. On POD \#12 an incidental segmental PE was discovered on follow-up imaging and required anticoagulation. The patient was discharged home on POD \#15 and continued to do well as of his most recent follow up two years postoperatively.

\section{Conclusion}

Open thoracoabdominal aortic aneurysm (TAAA) repair is a high-risk surgery associated with significant morbidity and mortality. Successful perioperative management requires a large team working together with a clear operative plan. Team leaders need to be aware of the numerous risks and implement strategies to mitigate these risks. We detail an off-pump perioperative approach involving retrograde aortic perfusion, transesophageal echocardiography, and multiple spinal cord protective strategies which resulted in a favorable patient outcome. 


\section{Conflicts of Interest}

The authors do not have any Conflict of interests.

\section{Acknowledgments}

None.

\section{Funding}

None.

\section{References}

1. Illig KA, Green RM, Ouriel K, et al. Primary fibrinolysis during supraceliac aortic clamping. J Vasc Surg. 1997;25(2):244-251.

2. Casati V, Sandrelli L, Speziali G, et al. Hemostatic effects of tranexamic acid in elective thoracic aortic surgery: a prospective, randomized, double-blind, placebo controlled study. J Thorac Cardiovasc Surg. 2002;123(6):1084-1091.

3. Day JR, Punjabi PP, Randi AM, et al. Clinical inhibition of the seventransmembrane thrombin receptor (PAR1) by intravenous aprotinin during cardiothoracic surgery. Circulation. 2004;110(17):2597-2600

4. Sedrakyan A, Treasure T, Elefteriades JA. Effect of aprotinin on clinical outcomes in coronary artery bypass graft surgery: a systematic review and meta-analysis of randomized clinical trials. $J$ Thorac Cardiovasc Surg. 2004;128(3):442-448.

5. Henry DA, Carless PA, Moxey AJ, et al. Anti-fibrinolytic use for minimising perioperative allogeneic blood transfusion. Cochrane Database Syst Rev. 2011;19(1):CD001886.

6. Carless PA, Moxey AJ, Stokes BJ, et al. Are anti fibrinolytic drugs equivalent in reducing blood loss and transfusion in cardiac surgery? A meta-analysis of randomized head-to-head trials. BMC Cardiovasc Disord. 2011;5:19.

7. Mangano DT, Tudor IC, Dietzel C. The risk associated with aprotinin in cardiac surgery. $N$ Engl J Med. 2006;354(4):353-365.

8. Karkouti K, Beattie WS, Dattilo KM, et al. A propensity score case-control comparison of aprotinin and tranexamic acid in hightransfusion-risk cardiac surgery. Transfusion. 2006;46(3):327-338.
9. Sedrakyan A, Treasure T, Elefteriades JA, et al. Effect of aprotinin on clinical outcomes in coronary artery bypass graft surgery: a systematic review and meta-analysis of randomized clinical trials. J Thorac Cardiovasc Surg. 2004;128(3):442-448.

10. Kahn RA, Stone ME, Moskowitz DM. Anesthetic Consideration for Descending Thoracic Aortic Aneurysm Repair. Semin Cardiothorac Vasc Anesth. 2007;11(3):205-223.

11. Money SR, Ballinger BA, Crockett DE, et al. The effects of supraceliac aortic clamping on the anticoagulant effects of heparin. The American Journal of Surgery. 1996;172(2):155-157.

12. Sabatini S, Kurtzman NA. Bicarbonate Therapy in Severe Metabolic Acidosis. JASN. 2009;20(4):692-695.

13. Wiederkehr M, Emmett M, Sterns RH, et al. Bicarbonate therapy in lactic acidosis. 2013.

14. Gehlbach BK, Schmidt GA. Bench-to-bedside review: Treating acidbase abnormalities in the intensive care - The role of buffers. Crit Care. 2004;8(4):259-265.

15. Fayad A, Yang $\mathrm{H}$, Nathan $\mathrm{H}$, et al. Acute diastolic dysfunction in thoracoabdominal aortic aneurysm surgery. Can J Anesth. 2006;53(2):168-173.

16. Fayad A, Sawchuk C, Yang H, et al. Transesophageal echocardiography in the management of left atrio-femoral bypass during thoracoabdominal aortic aneurysm repair: a case report. Can J Anesth. 2002;49(10):10811083 .

17. Hsu CCT, Kwan GNC, van Driel ML, et al. Distal aortic perfusion during thoracoabdominal aneurysm repair for prevention of paraplegia (Review). Cochrane Database Syst Rev. 2012;14(3):CD008197.

18. Madhusudan P, Wei L. Descending thoracic aortic aneurysms. Continuing Education in Anesthesia, Critical Care \& Pain. 2006;6(2):54-59.

19. Khan SN, Stansby G. Cerebrospinal fluid drainage for thoracic and thoracoabdominal aortic aneurysm surgery (Review). Cochrane Database Syst Rev. 2012;17(0):CD003635. 